\begin{tabular}{|c|l|}
\hline Title & A nalytic continuation of the Birkhoff-Rott equation in complex-time domain \\
\hline Author(s) & SA KA JO, TA KA SHI \\
\hline Citation & $\begin{array}{l}\text { European Journal of A pplied Mathematics, 15, 39-53 } \\
\text { https://doi.org/10.1017/S0956792503005230 }\end{array}$ \\
\hline Issue Date & 20040414 \\
\hline Doc URL & http://hdl.handle.net/2115/5432 \\
\hline Rights & Copyright $\odot 2004$ Cambridge University Press \\
\hline Type & article \\
\hline File Information & EJAM15-1.pdf \\
\hline
\end{tabular}

Instructions for use 


\title{
Analytic continuation of the Birkhoff-Rott equation in complex-time domain
}

\author{
TAKASHI SAKAJO \\ Graduate School of Mathematics, Nagoya University, Furo-cho Chikusa-ku Nagoya Aichi 464-8602, Japan
}

(Received 26 September 2001; revised 15 January 2003)

\begin{abstract}
A vortex sheet is a surface across which the velocity field of incompressible and inviscid flows has a jump discontinuity. Mathematical and numerical studies reveal that a two-dimensional vortex sheet, which is governed by the Birkhoff-Rott equation, acquires a singularity in finite time without forming rolling-up spiral. On the other hand, numerical computation of a regularized Birkhoff-Rott equation shows that the vortex sheet evolves into a rolling-up doubly branched spiral. Because of the finite-time singularity, it is impossible to regard the rolling-up spiral as a solution of the Birkhoff-Rott equation as long as time is real. However, it may be possible to analytically continue the equation to the spiral along a path to get around the singularity in complex-time plane. In the present article, we consider singularities in complex-time plane for the regularized Birkhoff-Rott equation by numerical means. Distribution of the complex singularities and their limiting behaviour indicate that it is absolutely impossible to perform analytic continuation in complex-time domain to the spiral solution. Furthermore, we propose a simple model of a doubly branched spiral and investigate it mathematically. The model is successful in approximating the rolling-up motion of the vortex sheet. Comparing the vortex-sheet motion with the model indicate that the doubly branched spiral with infinite windings at the centre could be a solution of the Birkhoff-Rott equation beyond the singularity time.
\end{abstract}

\section{Introduction}

A vortex sheet is a surface across which the velocity field of incompressible and inviscid fluids has a jump discontinuity. The study of vortex sheets contributes to an understanding of turbulent shear layers, since they approximate a coherent structure in an ideal flow with high-Reynolds number. The motion of two-dimensional vortex sheets is governed by an integro-differential equation, called the Birkhoff-Rott equation (Birkhoff [2]). While theoretical studies proved a short-time existence of an analytic solution [3, 4, 20], asymptotic analysis [11, 12] and numerical computations [9, 18] showed strong evidences that $2 \mathrm{D}$ vortex sheets lost analyticity in finite time.

Numerical computation of the Birkhoff-Rott equation is practically quite difficult, since not only do solutions blow up in finite time, but also linearization of the equation leads to an ill-posed problem in the sense of Hadamard due to the Kelvin-Helmholtz instability [15]. A treatment to avoid these numerical difficulties is regularization of the equation $[1,10]$. Krasny regularized the Birkhoff-Rott equation by introducing an artificial parameter $\delta$. Namely, let $(x(\Gamma, t), y(\Gamma, t))$ denote a two-dimensional vortex sheet, 
where $\Gamma$ is a Lagrangian parameter along the sheet and $t$ is time. A periodic boundary condition is imposed on the vortex sheet: $x(\Gamma+1, t)=x(\Gamma, t)+1$ and $y(\Gamma+1, t)=y(\Gamma, t)$. Then the Birkhoff-Rott equation is given by

$$
\begin{aligned}
& \frac{\partial x}{\partial t}=-\frac{1}{2} \int_{0}^{1} \frac{\sinh 2 \pi(y-\tilde{y})}{\cosh 2 \pi(y-\tilde{y})-\cos 2 \pi(x-\tilde{x})} d \tilde{\Gamma}, \\
& \frac{\partial y}{\partial t}=\frac{1}{2} \int_{0}^{1} \frac{\sin 2 \pi(x-\tilde{x})}{\cosh 2 \pi(y-\tilde{y})-\cos 2 \pi(x-\tilde{x})} d \tilde{\Gamma},
\end{aligned}
$$

where $\tilde{x}=x(\tilde{\Gamma}, t)$ and $\tilde{y}=y(\tilde{\Gamma}, t)$. Krasny proposed the following regularized equations:

$$
\begin{aligned}
& \frac{\partial x}{\partial t}=-\frac{1}{2} \int_{0}^{1} \frac{\sinh 2 \pi(y-\tilde{y})}{\cosh 2 \pi(y-\tilde{y})-\cos 2 \pi(x-\tilde{x})+\delta^{2}} d \tilde{\Gamma}, \\
& \frac{\partial y}{\partial t}=\frac{1}{2} \int_{0}^{1} \frac{\sin 2 \pi(x-\tilde{x})}{\cosh 2 \pi(y-\tilde{y})-\cos 2 \pi(x-\tilde{x})+\delta^{2}} d \tilde{\Gamma} .
\end{aligned}
$$

We call (1.1), (1.2) the $\delta$-equations. When $\delta$ is exactly zero, the $\delta$-equations are identical to the Birkhoff-Rott equation. By computing the $\delta$-equations for a given initial condition numerically, Krasny found that the regularized vortex sheet was smooth for all time and evolved into a rolling-up doubly branched spiral. Furthermore, investigating the limiting shape of the spiral as $\delta \rightarrow 0$, he discussed whether the spiral could be a solution of the Birkhoff-Rott equation after the singularity time.

However, there is a serious gap of analyticity: while the solution of the $\delta$-equations is smooth for all time, that of the Birkhoff-Rott equation is analytic only for a finite time and never evolves into the smooth rolling-up spiral. As long as the time variable is real, the solution of the Birkhoff-Rott equation cannot be analytically continued to the smooth spiral due to the singularity formation. However, it might be possible to choose a path to get around the singularity in the complex-time plane, along which we define the smooth roll-up solution by analytic continuation. In the present paper, we compute numerically all singularities of the $\delta$-equations in the complex-time plane, which we call complex singularity times. Thus, we consider their limiting behaviour as $\delta$ tends to zero, to discuss whether the smooth rolling-up solution could be an analytically continued solution of the Birkhoff-Rott equation.

The paper is organized as follows: in $\S 2$, we introduce a numerical method to search the complex singularity times. In $\S 3$, by using the numerical method, we obtain the distribution of the complex singularity times of the $\delta$-equations and investigate its behaviour as $\delta$ goes to zero. In $\S 4$, we propose a simple model to describe a rolling-up double branched spiral. By comparing the model spiral with the regularized vortex sheet, we show that the model is successful in approximating the rolling-up motion of the vortex sheet in complex-time domain. In addition, we discuss a solution of the Birkhoff-Rott equation beyond the singularity time. Finally, we give conclusions in $\S 5$.

\section{Numerical method}

We explain a numerical method to find complex singularity times. The numerical method was originally developed by Sulem et al. [19] to detect critical times when singularities 
appear in partial differential equations. The method has been successfully applied to the Birkhoff-Rott equation to investigate the singularity formation $[9,13,14,17,18]$. Here, we extend the method so that it can detect complex singularity times.

Extending $x(\Gamma, t)$ and $y(\Gamma, t)$ to complex-valued functions defined for $\Gamma \in \mathbf{R}$ and $t \in \mathbf{C}$, we regard the $\delta$-equations (1.1) and (1.2) as the equations for the four real-valued functions $x(\Gamma, t)=x_{r}(\Gamma, t)+\mathrm{i} x_{i}(\Gamma, t)$ and $y(\Gamma, t)=y_{r}(\Gamma, t)+\mathrm{i} y_{i}(\Gamma, t)$. The real parts of the initial conditions of $x$ and $y$ are the same as used by Krasny $[9,10]$. We set the imaginary parts of the initial conditions to zero. Namely,

$$
\left\{\begin{array}{llll}
x_{r}(\Gamma, 0) & =\Gamma & +0.01 \sin 2 \pi \Gamma, & x_{i}(\Gamma, 0)=0 \\
y_{r}(\Gamma, 0) & = & -0.01 \sin 2 \pi \Gamma, & y_{i}(\Gamma, 0)=0
\end{array}\right.
$$

These functions $x$ and $y$ are disctetized by $N=2048$ points. To compute the integrations of the right-hand sides of (1.1) and (1.2) numerically, we use the spectrally accurate method proposed by Shelley [18].

As for the temporal integration, we give an integration path in complex-time plane, along which the equations (1.1) and (1.2) are integrated temporally by using the fourthorder Runge-Kutta method. Note that there is no worry about the path chosen, since the temporal integration is independent of choice of the path as long as solutions are analytic. In addition, every time step of the temporal integration, Krasny's Fourier filtering technique is implemented to avoid accumulation of the round-off error $[6,9,18]$. For a detailed description of the filtering technique, refer to Krasny [9].

We explain how to find complex singularity times. Suppose that the functions $x(\Gamma, t)$ and $y(\Gamma, t)$ are represented by Fourier series every time step:

$$
x(\Gamma, t)=\Gamma+\sum_{k=-\frac{N}{2}}^{\frac{N}{2}-1} p_{k}(t) \mathrm{e}^{2 \pi \mathrm{i} k \Gamma}, y(\Gamma, t)=\sum_{k=-\frac{N}{2}}^{\frac{N}{2}-1} q_{k}(t) \mathrm{e}^{2 \pi \mathrm{i} k \Gamma} .
$$

Then we study the asymptotic behaviour of the spectra $p_{k}(t)$ and $q_{k}(t)$; we use an ansatz for their asymptotic decay. Assume that the leading terms of the spectra become

$$
\begin{aligned}
& \left|p_{k}(t)\right| \sim C_{p} k^{-\beta_{p}}\left(1+D_{p} \frac{1}{k}\right) \exp \left(-\alpha_{p}(t) k\right), \\
& \left|q_{k}(t)\right| \sim C_{q} k^{-\beta_{q}}\left(1+D_{q} \frac{1}{k}\right) \exp \left(-\alpha_{q}(t) k\right) .
\end{aligned}
$$

This ansatz is the same as Shelley's, which are derived from Moore's asymptotic analysis of the vortex sheet. We compute the values of $C_{p}, D_{p}, \alpha_{p}$ and $\beta_{p}$ by requiring that (2.2) holds pointwise at consecutive four points of $p_{i}$ for $i=k, k+1, k+2$ and $k+3$. In the same way, the values of $C_{q}, D_{p}, \alpha_{q}$ and $\beta_{q}$ are computed by the fit to $q_{k}(t)$. Note that, as shown in Shelley [18], if the numerical computation is sufficiently accurate, these values are determined independently of $k$ when the integration path approaches the singularity time. To achieve accurate computation this time, we implemented all the numerical procedures with 128 bit floating-point numbers. If either $\alpha_{p}$ or $\alpha_{q}$ is close to zero, we estimate the time when it vanishes by extrapolation. This is the critical time when $x(\Gamma, t)$ or $y(\Gamma, t)$ 


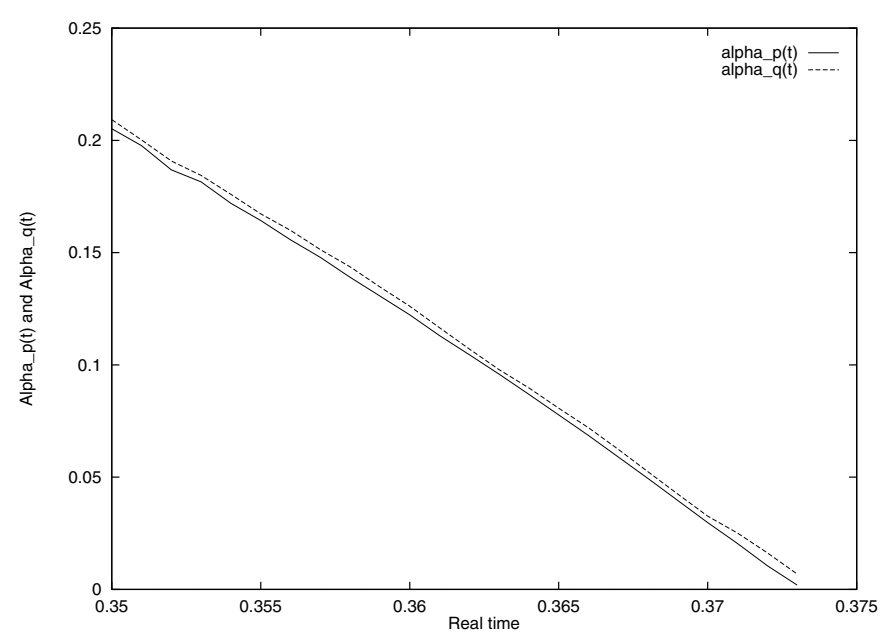

FIGURE 1. The fits to $\alpha_{p}$ and $\alpha_{q}$ as functions of the real time, when $\delta=0$ and the integration path is the real-time axis.

loses its analyticity, since at that time $|x(\Gamma, t)|$ or $|y(\Gamma, t)|$ is expressed asymptotically as

$$
|x(\Gamma, t)| \sim \Gamma+\sum_{k}\left|C_{p}\right||k|^{-\beta_{p}} \quad \text { or } \quad|y(\Gamma, t)| \sim \sum_{k}\left|C_{q}\right||k|^{-\beta_{q}}
$$

We give an example showing that the method works well, in which we apply the method to the Birkhoff-Rott equation; when the regularization parameter $\delta$ is zero and an integration path goes along the real-time axis. Figure 1 shows the fits to $\alpha_{p}$ and $\alpha_{q}$ as functions of the real time. The value of $\alpha_{p}$ monotonically decreases towards zero and is estimated to be zero at $t \approx 0.374+0 \mathrm{i}$ by extrapolation. The critical time is equal to that Krasny [9] estimated up to the second digit. In this paper, the critical time is denoted specifically by $t_{c}$.

\section{Complex singularity times of the $\delta$-equations}

\subsection{Distribution of complex singularity times when $\delta=0.1$}

Figure 2 shows the distribution of complex singularity times of the $\delta$-equations in the complex-time plane. The regularization parameter $\delta$ is 0.1 . The complex singularity times exist continuously on the boundaries between gray regions and the white region. At each complex singularity time, the solution of the $\delta$-equations loses analyticity for some real $\Gamma$. The distribution is symmetric with respect to the real-time axis. Because of the continuity of the distribution, we can define no analytic solution inside the gray regions by analytic continuation for any integration path starting from the origin. On the other hand, if we take an integration path along the real-time axis, we define an analytic solution along the path by analytic continuation, since there is no complex singularity time in the path. This is why Krasny's regularization works well to compute the $\delta$-equations numerically. 


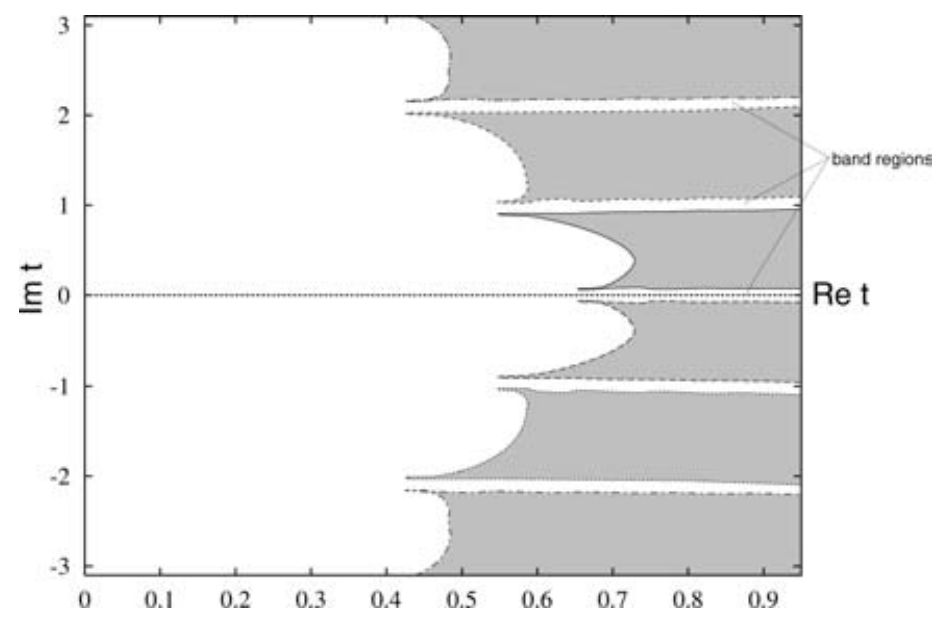

FIGURE 2. Distribution of the complex singularity times of the $\delta$-equations when $\delta=0.1$. The complex singularity times are on the boundaries between gray regions and the white region.

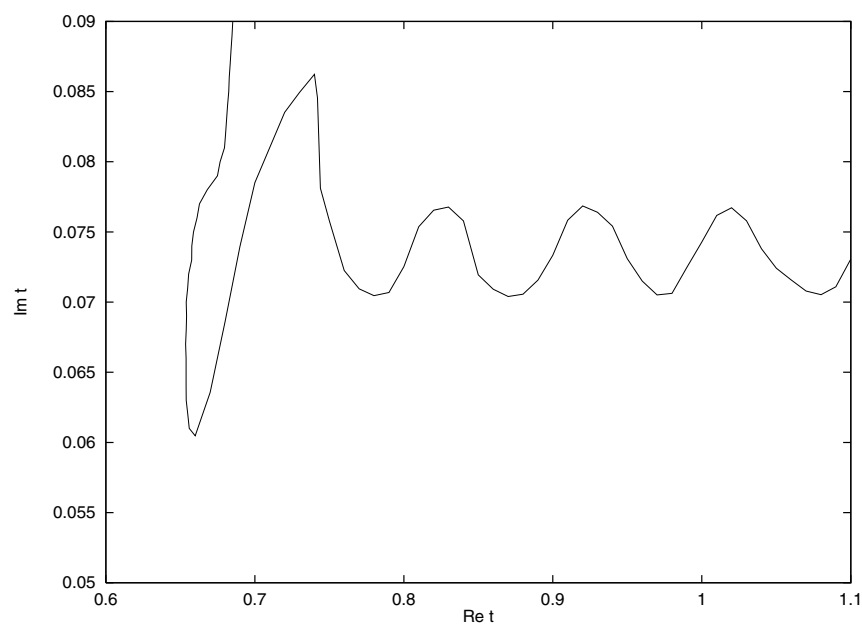

FIGURE 3. Distribution of the complex singularity times in the neighbourhood of the real-time axis. They are distributed approximately periodically in the real-time direction after $\operatorname{Re} t \approx 0.85$.

Now, let us describe the distribution precisely; there are narrow band-like white regions between the gray regions. The band regions exist periodically in the imaginary time direction. In Figure 3, we re-plot the distribution in the neighbourhood of the realtime axis. The figure also shows that the distribution is approximately periodic in the real-time direction beyond $\operatorname{Re} t \approx 0.85$. We observe the same periodic structure for other band regions. It indicates that the complex singularity times are approximately distributed doubly periodically in the band regions.

Next, we examine the relation between the distribution and evolution of the vortex sheet. Figure 4 shows an evolution of the vortex sheet when an integration path is taken 


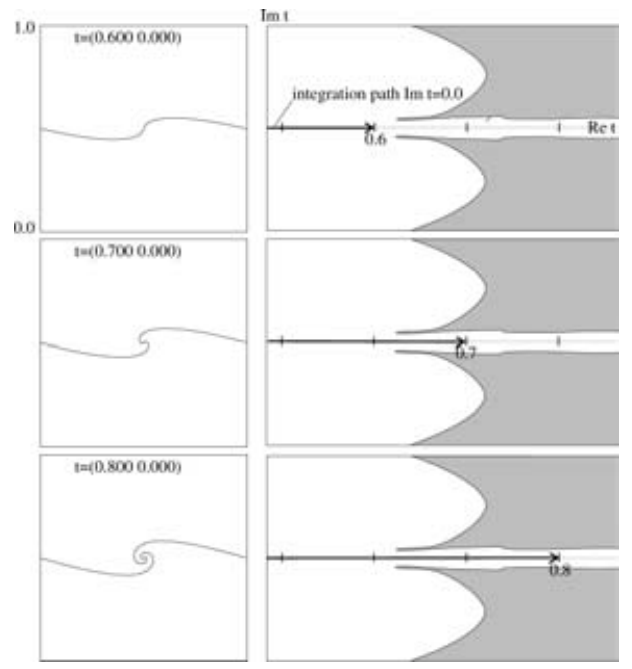

Figure 4. (Left) Numerical solution of the $\delta$-equations when $\delta=0.1$. (Right) Integration path, which is taken along the real-time axis.

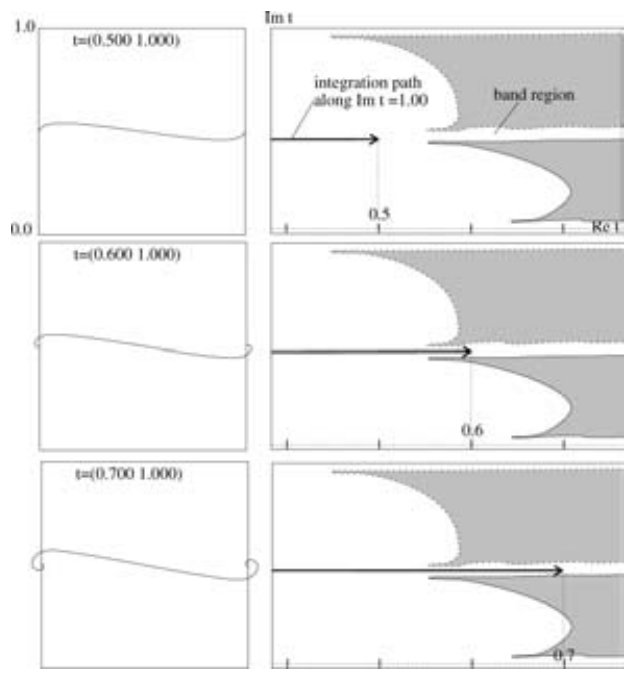

Figure 5. (Left) Numerical solution of the $\delta$-equations when $\delta=0.1$. (Right) Integration path, which is taken along $\operatorname{Im} t=1$.

along the real-time axis. When the path enters the band region, i.e. $\operatorname{Re} t>0.75$, the vortex sheet begins rolling up in the middle. Figure 5 shows an another example when we take an integration path along $\operatorname{Im} t=1$. The vortex sheet also rolls up at the boundary after the path gets into the band region. As for the other band regions, we can verify that the vortex sheet likewise rolls up when the integration path gets into the band regions. These numerical results indicate that there is a close relation between the doubly periodic band structure of complex singularity times and the rolling-up motion of the vortex sheet. 


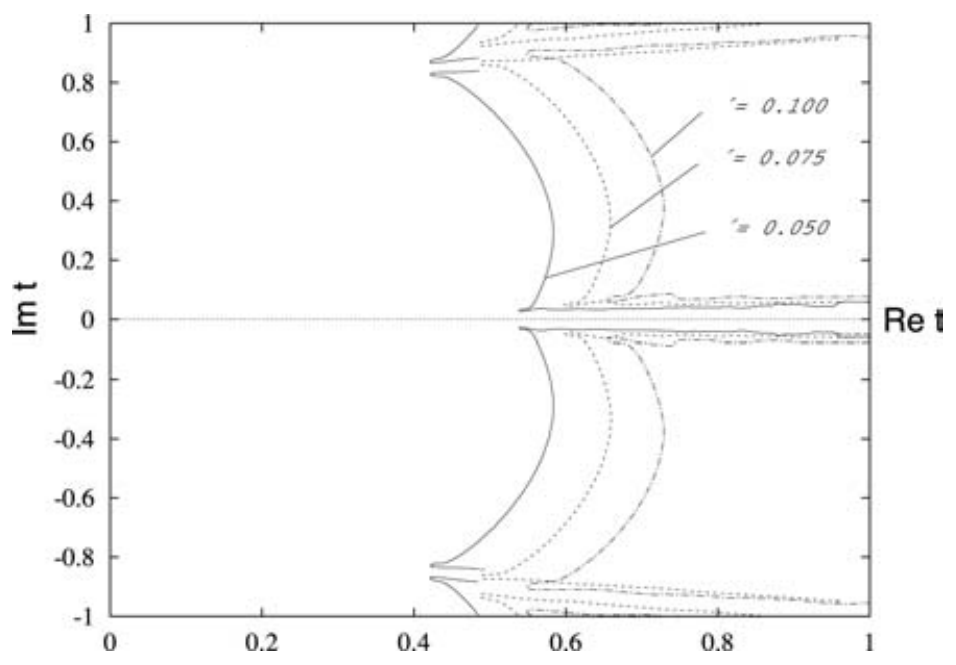

FIGURE 6. Distributions of complex singularity times in the neighbourhood of the real-time axis for various $\delta$.

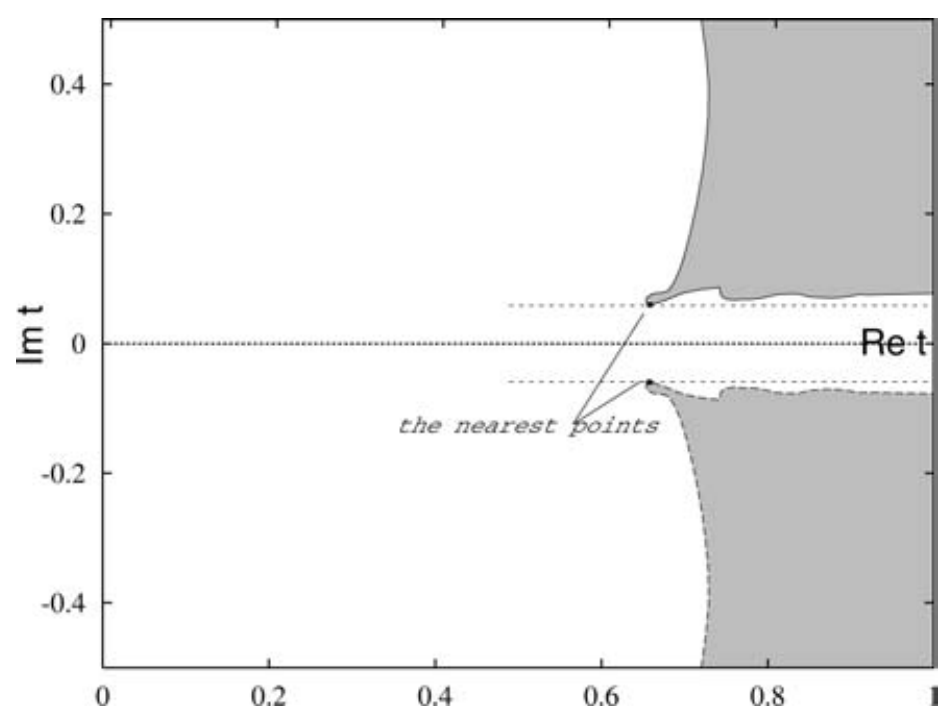

FIGURE 7. Position of the complex singularity times that are the nearest to the real-time axis when $\delta=0.1$. They are located at the tips of the band region.

\subsection{Behaviour of the distribution as $\delta \rightarrow 0$}

We study how the distribution of the complex singularity times behaves when $\delta \rightarrow 0$. Figure 6 shows distributions in the neighbourhood of the real-time axis for $\delta=0.05,0.075$ and 0.1 . While the distribution moves to the left, the width of the band regions gets smaller as $\delta \rightarrow 0$.

To see how the band regions behave, we focus on two complex singularity times that are the nearest to the real-time axis, located at the tips of the distribution; see Figure 7. 


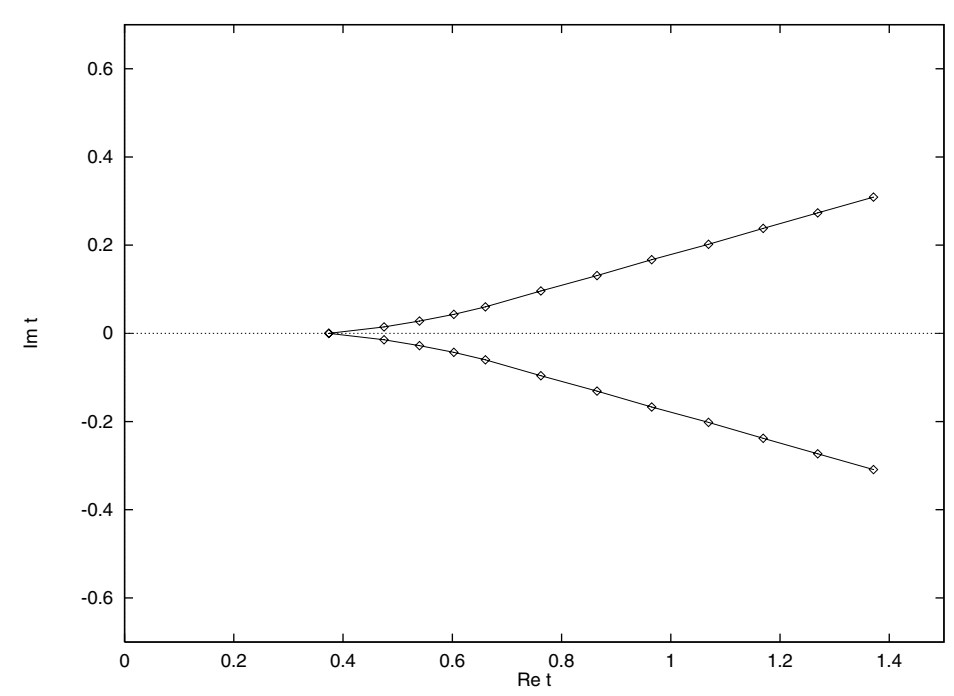

FIGURE 8. Positions of the pair of the complex singularity times which are the nearest to the real-time axis for $\delta=0,0.025,0.05,0.075,0.1,0.15,0.2,0.25,0.3,0.35,0.4$ and 0.45 from the left. They converge at the critical time $t_{c}$ of the Birkhoff-Rott equation in the real-time axis.

They are complex conjugate with each other because of the symmetry of the distribution. We plot the two nearest complex singularity times in Figure 8 when $\delta$ decreases from 0.45 to 0 . The pair of the nearest complex singularity times approach each other as $\delta$ tends zero and then collide at the singularity time $t_{c}$ in the real-time axis. This means that the entrance of the band region is blocked at $t_{c}$. Hence, it is impossible to define an analytic solution of the Bikhoff-Rott equation beyond $t_{c}$ by analytic continuation along the real-time axis. This is why the solution of the Birkhoff-Rott equation blows up in finite time, although the solution of the $\delta$-equations is smooth for all time. Furthermore, as for the another band regions, the widths of their entrances get smaller when $\delta$ tends to zero and likewise close when $\delta$ is exactly zero. Consequently, no integration path starting from the origin can enter the band regions, which means that the Birkhoff-Rott equation cannot be analytically continued to the smooth rolling-up spiral not only along the real time axis but also along any path in the complex-time domain.

\section{Model of a rolling-up double branched spiral}

We propose a simple model of a rolling-up double branched spiral. The model is based on an observation of the rolling-up motion of the vortex sheet; the vorticity shifts to the centre of the spiral and forms a high-vorticity region in the centre while the vortex sheet rolls up. This is our model: for a given curve with the periodic boundary condition, we assume that a point vortex is located in the middle of the curve and passive scalars are distributed uniformly in the curve except of the centre. Since this model approximates the rolling-up vortex sheet motion, the analysis of the model gives more information about the sturucture of complex singularity times and its limiting behaviour of the vortex sheet problem. 
(a) Vortex sheet

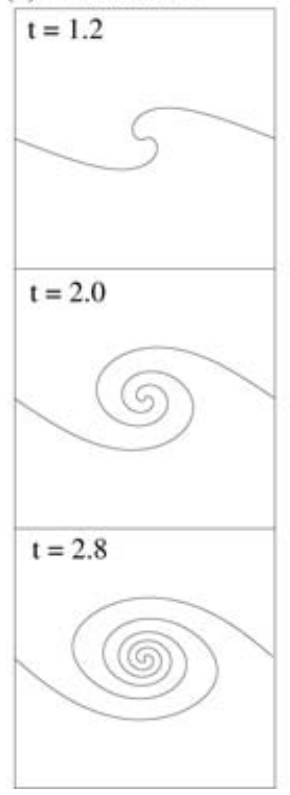

(b) Model spiral

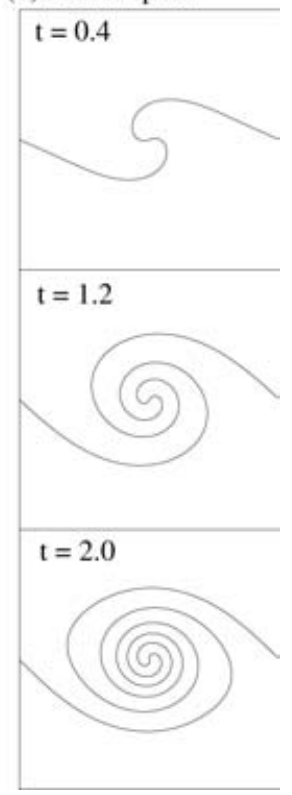

FIGURE 9. (a) Numerical solution of the $\delta$-equations for $\delta=0.3$. (b) Numerical solution of the model equation for $\delta=0.6$. These evolutions look similar qualitatively except the size of the spiral.

\subsection{Equation and its solution}

We investigate a regularized equation of the passive scalars advected by the point vortex. We assume that the point vortex is located at the origin and the passive scalars are distributed in the segment $[-0.5,0.5)$ at the beginning. That is,

$$
x(\Gamma, 0)=-0.5+\Gamma, y(\Gamma, 0)=0, \quad(0 \leqslant \Gamma<1, \Gamma \neq 0.5) .
$$

Then, while the point vortex is steady for all time, the regularized equation of the passive scalars at $(x(\Gamma, t), y(\Gamma, t))$ are described by

$$
\frac{\mathrm{d} x}{\mathrm{~d} t}=-\frac{1}{2} \frac{\sinh 2 \pi y}{\cosh 2 \pi y-\cos 2 \pi x+\delta^{2}}, \quad \frac{\mathrm{d} y}{\mathrm{~d} t}=\frac{1}{2} \frac{\sin 2 \pi x}{\cosh 2 \pi y-\cos 2 \pi x+\delta^{2}},
$$

whose initial conditions are given by (4.1). We compare evolutions of the passive scalars and the vortex sheet. Figure 9 shows a numerical solution of the vortex sheet from $t=1.2$ when $\delta=0.3$ and that of the passive scalars from $t=0.4$ when $\delta=0.6$. Although the starting times of these evolutions are different, the time interval between each figures are the same and the winding number of the spirals looks similar. The comparison tells us that the model approximates qualitatively the evolution of the rolling-up motion of the vortex sheet when $\delta$ is chosen properly.

Another advantage in considering the model equation is that it can be solved explicitly. The denominator of the right-hand side of the equations (4.2), which is denoted by 
$K_{\delta}(\Gamma, t)$, is invariant in time because $\frac{d K_{\delta}}{d t}=0$. Hence, we define $K_{\delta}$ by

$$
K_{\delta}(\Gamma, t)=K_{\delta}(\Gamma, 0)=2\left(1+\cos 2 \pi \Gamma+\delta^{2}\right) \equiv K_{\delta}(\Gamma) .
$$

Introducing a new time variable $\tau=\frac{2 \pi t}{K_{\delta}(\Gamma)}$ and space variables $X=\sin 2 \pi x, Y=$ $\cos 2 \pi x, P=\sinh 2 \pi y$ and $Q=\cosh 2 \pi y$, we rewrite the equation (4.2) as follows:

$$
\frac{\mathrm{d} Y}{\mathrm{~d} \tau}=X P, \quad \frac{\mathrm{d} Q}{\mathrm{~d} \tau}=X P .
$$

Initial conditions of the variables are $X(\Gamma, 0)=-\sin 2 \pi \Gamma, \quad Y(\Gamma, 0)=-\cos 2 \pi \Gamma$, $P(\Gamma, 0)=0$ and $Q(\Gamma, 0)=1$, respectively. It follows from (4.3) that $Y-Q$ is independent of $\tau$. Accordingly, we also define an another invariant, $A(\Gamma)$, by

$$
Y(\Gamma, \tau)-Q(\Gamma, \tau)=Y(\Gamma, 0)-Q(\Gamma, 0)=-(1+\cos 2 \pi \Gamma) \equiv A(\Gamma) .
$$

We obtain $Y(\Gamma, \tau)=Q(\Gamma, \tau)+A(\Gamma)$. Note that since $0 \leqslant \Gamma<1$ and $\Gamma \neq 0.5,-2 \leqslant$ $A(\Gamma)<0$. On the other hand, there are relations between the space variables; $X^{2}+Y^{2}=1$ and $Q^{2}-P^{2}=1$. Substituting them into (4.3), we obtain the equation only for $Q$ :

$$
\left(\frac{\mathrm{d} Q}{\mathrm{~d} \tau}\right)^{2}=-\left(1-(Q+A)^{2}\right)\left(1-Q^{2}\right)
$$

The solution of this equation is an elliptic function. If we put $Q=\frac{1}{Q_{1}}+1$, then (4.4) becomes

$$
\left(\frac{\mathrm{d} Q_{1}}{\mathrm{~d} \tau}\right)^{2}=-2 A(A+2) Q_{1}^{3}-\left(A^{2}+6 A+4\right) Q_{1}^{2}-2(A+2) Q_{1}-1,
$$

the solution of which is

$$
Q_{1}(A, \tau)=-\frac{2}{A(A+2)}\left(\wp(\tau)+\frac{A^{2}+6 A+4}{12}\right) .
$$

The function $\wp(\tau)$ is Weierstrass's elliptic function, which is an elliptic function with index two. Since $\tau=\frac{2 \pi t}{K_{\delta}}$ and $K_{\delta}(\Gamma)=2\left(\delta^{2}-A(\Gamma)\right)$, the function $Q$ is given explicitly as a function of $A$ and $t$ by

$$
Q(A, t)=1-\frac{6 A(A+2)}{12 \wp\left(\frac{\pi t}{\delta^{2}-A}\right)+A^{2}+6 A+4} .
$$

The explicit representation of the solution $Q$ makes us possible to consider complex singularity times of $\mathrm{Q}$, which satisfy the following equation:

$$
12 \wp\left(\frac{\pi t}{\delta^{2}-A}\right)+\left(A^{2}+6 A+4\right)=0 .
$$

According to the properties of $\wp$ function, for fixed $\delta \neq 0$ and $A \neq 0$, there are two solutions of (4.5) in a fundamental period-parallelogram of the $\wp$-function, which are symmetric with respect to the pole. Furthermore, they are distributed doubly periodically in whole complex-time plane. Hence, when solving the equation (4.5) for all $A$, we obtain doubly periodic band-like sequences of the complex singularity times of $Q$. This explains 
qualitatively the numerical results in $\S 3$ that the evolution of the rolling-up vortex sheet is closely related to the doubly periodic band structure of the complex singularity times in both the real-time and the imaginary-time directions.

\subsection{Behaviour of the complex singularity times as $\delta \rightarrow 0$}

We are interested in a singular behaviour such as collision of the complex singularity times of the model as $\delta \rightarrow 0$. When $A \neq 0$ and $\delta=0$, the complex singularity times satisfy the following equation:

$$
12 \wp\left(-\frac{\pi t}{A}\right)+\left(A^{2}+6 A+4\right)=0 .
$$

Although there are two solutions of this equation, they never collide. Hence, no singular behaviour is observed in this case. Now, we consider behaviour of the complex singularity times when $A=0$. Since the function $Q$ is undefined at $A=0$, we define the function $Q(0, t)$ as the limit value of $Q(A, t)$ when $A \rightarrow 0$ for $\delta \neq 0$, and then observe how the complex singularity times behave as $\delta \rightarrow 0$.

- Continuity of $Q$ at $A=0$ : As $A \rightarrow 0, Q(0, t) \rightarrow 1$ for all $t$. Hence, $y(0.5, t)=0$ for all time.

- First derivative of $Q$ with respect to $\Gamma$ at $A=0$ :

$$
\frac{\mathrm{d} Q}{\mathrm{~d} \Gamma}=\frac{\mathrm{d} Q}{\mathrm{~d} A} \cdot \frac{\mathrm{d} A}{\mathrm{~d} \Gamma}=\frac{\mathrm{d} Q}{\mathrm{~d} A} \cdot 2 \pi \sin 2 \pi \Gamma .
$$

Substituting $A=0$ into it, we obtain $\frac{d Q}{d \Gamma}(0, t)=0$ for all $t$.

- Second derivative of $Q$ with respect to $\Gamma$ at $A=0$ : Since

$$
\frac{\mathrm{d}^{2} Q}{\mathrm{~d} \Gamma^{2}}=\frac{\mathrm{d}^{2} A}{\mathrm{~d} \Gamma^{2}} \cdot \frac{\mathrm{d} Q}{\mathrm{~d} A}+\frac{\mathrm{d}^{2} Q}{\mathrm{~d} A^{2}} \cdot\left(\frac{\mathrm{d} A}{\mathrm{~d} \Gamma}\right)^{2},
$$

the second derivative of $Q$ at $A=0$ is given by

$$
\frac{\mathrm{d}^{2} Q}{\mathrm{~d} \Gamma^{2}}(0, t)=\frac{12 \pi^{2}}{3 \wp\left(\frac{\pi t}{\delta^{2}}\right)+1} .
$$

Since $Q(0, t)$ and its first derivative are constant for all time, it is the second derivative of $Q$ that can show singular behaviour when $\delta \rightarrow 0$. Hence, we study the complex singularity times of the second derivative. First, we consider an equation for complex $\tau$ :

$$
\wp(\tau)=-\frac{1}{3}
$$

There are two symmetric solutions in a fundamental period-parallelogram, which are distributed doubly periodically in the complex $\tau$-plane. Then mapping these complex singularity times into the complex $t$-plane by the scaling relation between $t$ and $\tau$, i.e. $\tau=\frac{\pi t}{\delta^{2}}$, we find that the complex singularity times as well as the period-parallelogram contracts to the origin in complex $t$-plane when $\delta$ tends to zero. Hence, when $\delta$ is exactly 

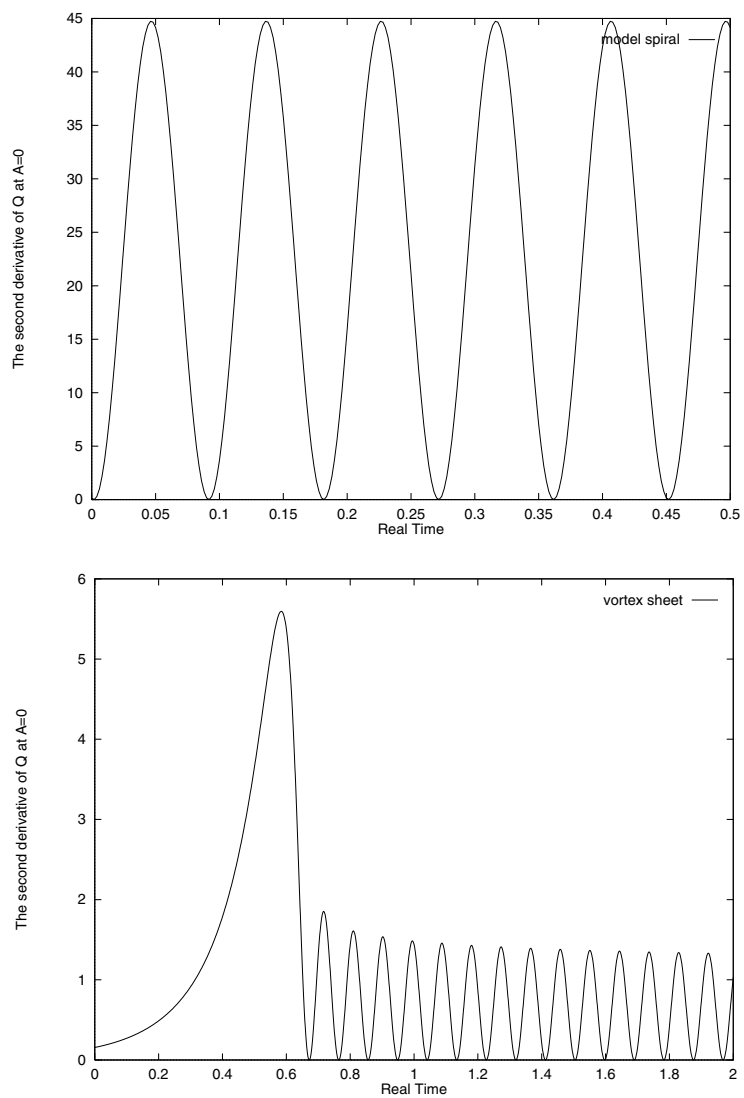

Figure 10. Evolution of the second derivative of $Q$ with respect to $\Gamma$ at $A=0(\Gamma=0.5)$. (Upper): Plot for the model spiral $(\delta=0.3)$. (Lower): Plot for the vortex sheet $(\delta=0.1)$.

zero, all the complex singularity times with $A=0$ concentrate into the origin, which is a quite singular behaviour.

We verify that the quadratic contracting behaviour is observed in the vortex sheet motion. Figure 10 shows the second derivative of the model $(\delta=0.3)$ and of the vortex sheet $(\delta=0.1$ ). While the second derivative of the model just changes periodically, that of the vortex sheet increases at the beginning, then decreases, and finally begins oscillating at $t \approx 0.7$, after which the vortex sheet rolls up. These figures indicate that the model approximates the rolling-up motion of the vortex sheet only after $t \approx 0.7$. Here, we pay attention to the wavelength of the second derivative while the vortex sheet rolls up. This is because the wavelength corresponds to one of the periods of the doubly periodic function (4.6). Figure 11 shows the periods of the second derivative of the model $(\times$ marks $)$ and of the vortex sheet $(+$ marks) for various $\delta$. Dotted lines in the figure are computed by a least-squares fit to these data. The period of the model decreases as $\delta^{2}$. As for the period of the vortex sheet, we fit the data to three types of functions: (a) $a_{1} \delta^{3}+a_{2} \delta^{2}+a_{3} \delta+a_{4}$, (b) $b_{1} \delta^{2}+b_{2} \delta+b_{3}$ and (c) $c_{1} \delta^{2}+c_{2} \delta$. Table 1 shows these fitting functions and their least square errors. The least square fit (a) indicates that the period of the vortex sheet decreases like a quadratic function rather than a cubic function. This agrees with the 




Figure 11. Periods of the second derivative of $Q$ for various $\delta$. The + marks represent the periods for the vortex sheet. The $\times$ marks show the periods for the model. The dotted curves are computed by the fit to these data.

Table 1. The fitting functions to the data of periods of the second derivative of $Q$ for the vortex sheet obtained by the least square s method and errors

\begin{tabular}{ccc}
\hline \hline Type & Fitted function & Error \\
\hline (a) & $-0.0001 \delta^{3}+1.0579 \delta^{2}+0.8346 \delta+0.0034$ & $9.658 \mathrm{e}-06$ \\
(b) & $1.0273 \delta^{2}+0.8578 \delta+0.0001$ & $1.037 \mathrm{e}-05$ \\
(c) & $1.0252 \delta^{2}+0.8389 \delta$ & $1.037 \mathrm{e}-05$ \\
\hline
\end{tabular}

model. Then, since the least square errors of the fits (b) and (c) become the same order, it is impossible to determine which fit is appropriate for the data. However, the periods approach to almost zero when $\delta \rightarrow 0$ as that of the model does.

\subsection{On a solution of the Birkhoff-Rott equation beyond the singularity time}

The period of the second derivative of $Q$ when $\delta=0$ suggests how a solution of the Birkhoff-Rott equation looks beyond the singularity time: the second derivative of $Q$ at $A=0$ is equivalent to square of the first derivative of $y$ at the centre of the spiral, namely

$$
\frac{\mathrm{d}^{2} Q}{\mathrm{~d} \Gamma^{2}}(0, t)=\left.\left(\frac{\mathrm{d}}{\mathrm{d} \Gamma}\right)^{2} \cosh 2 \pi y(\Gamma, t)\right|_{\Gamma=0.5}=\left(2 \pi \frac{\mathrm{d} y}{\mathrm{~d} \Gamma}(0, t)\right)^{2}
$$

Therefore, the periodic behaviour of the second derivative indicates that the $y$-component of the tangent vector to the doubly branched spiral at the centre changes periodically, which represents the rotation of the spiral. Furthermore, the period corresponds to how fast the doubly branched spiral rotates. That means, the smaller the period becomes, the 
faster the spiral rotates. According to the fit to the period of the second derivative of the model in Figure 11, the period becomes zero when $\delta=0$, which means that the spiral rotates with infinite speed. On the other hand, since the period of the vortex sheet almost tends to zero as $\delta$ goes to zero, the solution of the regularized Birkhoff-Rott equation approaches to a doubly branched spiral with very large (or possibly infinite) windings.

In the present numerical computation, it is hard to decide whether these quantities are exactly zero when $\delta=0$ because it is hard to resolve the data by the fit more accurately than the accuracy of the numerical computation. However, both quantities are very close to zero when $\delta=0$, which supports that a doubly branched spiral with infinite windings is the solution of the Birkhoff-Rott equation beyond the singularity time.

\section{Conclusions and discussions}

We investigated numerically complex singularity times when a solution of the $\delta$-equations lost its analyticity by regarding time variable as complex number. We gave distribution of the complex singularity times in complex-time plane, according to which they are distributed on the band like sequence that are doubly periodically in the imaginary-time and the real-time directions, while the vortex sheet is rolling up. Since the real-time axis is put between the structure, we define an analytic solution of the $\delta$-equations by analytic continuation. This is why numerical solutions of the $\delta$-equations is smooth for all time. We also pointed out a relation between the doubly periodic band-like structure of the complex singularity times and the rolling-up motion of the vortex sheet.

Then, we studied the behaviour of the distribution as $\delta$ approaches zero. The width of the band regions are getting smaller. When we observe carefully the complex singularity times that are the nearest to the real-time axis, they get closer to each other and collide at the singularity time of the Birkhoff-Rott equation when $\delta=0$. That means that the entrance of the band region is blocked at $t=t_{c}$. Therefore, we can define no analytic solution of the Birkhoff-Rott equation beyond $t_{c}$ into the band region by analytic continuation along the real-time axis. This is the reason why the solution of the BirkhoffRott equation blows up in finite time, although the solution of the $\delta$-equations is smooth for all time. In addition, since the entrances of the other band regions close as well, it is impossible to continue analytically the Birkhoff-Rott equation to smooth rolling-up solutions of the $\delta$-equations in the complex-time domain.

We proposed a simple model that describes a rolling-up doubly branched spiral. The model approximates the motion of the regularized vortex sheet qualitatively well after it begins rolling up. It explains the relation between the doubly periodic band structure of complex singularity times and the rolling-up doubly branched spiral. Comparison of the limiting behaviour of the complex singularity times of the vortex sheet with that of the model when $\delta \rightarrow 0$ implies that the solution of the regularized Birkhoff-Rott equation tends to the doubly branched spiral with infinite windings. Finally, let us note that the model explains the motion of the regularized vortex sheet well only during the intermediate time interval when the doubly branched vortex sheet is simply rotating. The model can't reveal both the initial evolution of the vortex sheet before it begins rolling up and a long-time evolution like what Sakajo \& Okamoto [16] computed. 


\section{Acknowledgements}

I am grateful to Professor Y. Kimura for discussion and advice. I thank Professor H. Okamoto and Professor Caflisch for giving me useful comments. The author is supported by the Grant-in-Aid for Scientific Research from the Japan Society for Promotion of Science.

\section{References}

[1] BAKer, B. R. \& Shelley, M. J. (1986) Boundary integral techniques for multi-connected domains. J. Comput. Phys. 64, 112-32.

[2] Birkhoff, G. (1962) Helmholtz and Taylor instability. Symp. Appl. Math. XII, pp. 55-76. AMS.

[3] Caflisch, R. E. \& Orellana, O. F. (1986) Long time existence for a slightly perturbed vortex sheet. Comm. Pure Appl. Math. 39, 807-38.

[4] Caflisch, R. E. \& Orellana, O. F. (1989) Singular solutions and ill-posedness for the evolution of vortex sheets. SIAM J. MaTH. ANAL. 20, 293-307.

[5] Diperna, R. \& Majda, A. (1987) Concentrations in regularizations for 2-d incompressible flow. Comm. Pure Appl. Maths. 40, 301-45.

[6] Ely, J. S. \& BaKer, G. R. (1993) High-precision calculations of vortex sheet motion. J. Comput. Phys. 111, 275-81.

[7] Kimura, Y. (1991) Vortex collapse from viewpoint of complex-time singularity. Physica D, 51, 512-19.

[8] Kimura, Y. (1995) Dynamics of complex singularities for Burger's equation. In: Makhankov, V. G., Bishop, A. R. and Holm, D. D. (eds.), Proc. 10th Int. Conf. on 'Nonlinear Systems \& Dynamical Systems', pp. 86-93. Los Alamos, CA. World Scientific.

[9] Krasny, R. (1986) A study of singularity formation in a vortex sheet by the point-vortex approximation. J. Fluid Mech. 167, 65-93.

[10] Krasny, R. (1986) Desingularization of periodic vortex sheet roll-up. J. Comput. Phys. 65, 292-313.

[11] Meiron, D. I., Baker, G. R. \& Orszag, S. A. (1982) Analytic structure of vortex sheets dynamics. Part I. Kelvin-Helmholtz instability. J. Fluid Mech. 113, 283-98.

[12] Moore, D. W. (1979) The spontaneous appearance of a singularity in the shape of an evolving vortex sheet. Proc. R. Soc. Lond. 365, 105-19.

[13] NiE, Q. \& BAKER, G. R. (1998) Application of adaptive quadrature to axisymmetric vortex sheet motion. J. Comput. Phys. 143, 49-69.

[14] Nitsche, M. (2001) Singularity formation in a cylindrical and a spherical vortex sheet. J. Comput. Phys. 143, 208-30.

[15] Saffman, P. G. (1992) Vortex Dynamics. Cambridge University Press.

[16] Sakajo, T. \& Окамото, H. (1998) An application of Draghicescu's fast summation method to vortex sheet motion. J. Phys. Soc. Japan, 62, 462-70.

[17] Sakajo, T. (2002) Formation of curvature singularity along vortex line in an axisymmetric, swirling vortex sheet. Phys. of Fluids, 14, 2886-2897.

[18] Shelley, M. J. (1992) A study of singularity formation in vortex-sheet morion by a spectrally accurate vortex method. J. Fluid Mech. 244, 493-526.

[19] Sulem, C., Sulem, P. L. \& Frisch, H. (1983) Tracing complex singularities with spectral methods. J. Comput. Phys. 50, 138-61.

[20] Sulem, C., Sulem, P. L., Bardos, C. \& Frisch, U. (1981) Finite time analyticity for the two and three dimensional Kelvin-Helmholtz instability. Comm. Math. Phys. 80, 485-516. 